\title{
CARACTERIZAÇÃO ISOTÓPICA DE ÁGUAS DE FORMAÇÃO HIPERSALINAS DE UM CAMPO DE PETRÓLEO DA BACIA DO RECÔNCAVO, BRASIL
}

\author{
Danilo Ribeiro de Sá Teles ${ }^{1}$, Antônio Expedito Gomes de Azevedo ${ }^{1}$ e Christian Pereira Lopes dos Santos ${ }^{2}$
}

Recebido em 21 dezembro, 2009 / Aceito em 15 março, 2010

Received on December 21, 2009 / Accepted on March 15, 2010

\begin{abstract}
Formation water is the water present in reservoir rock pores, produced together with petroleum. Usually it is hypersaline with concentrations that can be higher than $250 \mathrm{~g} / \mathrm{l}$ of dissolved solids. The concentration of the dissolved elements and the isotopic composition of the water and of some of the dissolved elements can be excellent tracers to study the dynamic of underground fluid flux, the mixing of distinct sources and the origin of salinization of these waters. This work reports the isotopic composition $\left(\delta^{2} \mathrm{H}, \delta^{18} 0\right)$ and electrical conductivity $(\mathrm{EC})$ of formation waters from 10 wells of a petroleum field of the Recôncavo Basin, looking for their evolution, interaction with the minerals and rocks and reservoir interconnection. The samples have electric conductivity ranging from 84 to $137 \mathrm{mS} / \mathrm{cm}$, with conductivity increasing with depth of production zone. It is observed an enrichment of deuterium and oxygen-18 with the depth of production zone, probably due to water-rock interactions that were probably increased by higher temperatures of the deeper levels and longer residence times. The samples from the production zone between 1450 to $1520 \mathrm{~m}$, drained by 7 of the 10 wells sampled, show a small range in isotopic composition and in electric conductivity, indicating homogeneity in this level of the reservoir. In the shallow level of $450 \mathrm{~m}$ the values of $\delta^{2} \mathrm{H}, \delta^{18} 0$ and $\mathrm{EC}$ are lower, with isotopic composition similar to the oceanic water, possibly representing the original water that entered the reservoir during the sedimentation of the basin.
\end{abstract}

Keywords: isotopic geophysics, formation water, stable isotopes, Recôncavo Basin, cryogenic distillation.

RESUMO. Água de formação é a água que ocorre naturalmente nos poros de uma rocha e é produzida junto com o petróleo. É geralmente hipersalina podendo chegar a concentrações de até $250 \mathrm{~g} / \mathrm{l}$ de sólidos dissolvidos. A concentração dos materiais nela dissolvidos e a composição isotópica da água e de alguns dos elementos nela presentes se constituem em excelentes traçadores, podendo revelar a dinâmica do fluxo subterrâneo, a mistura de fontes distintas e a origem da salinização dessas águas. Este trabalho reporta a composição isotópica $\left(\delta^{2} \mathrm{H}, \delta^{18} 0\right)$ e a condutividade elétrica (CE) de amostras de água de formação, provenientes de 10 poços de um campo de petróleo da Bacia do Recôncavo, com o objetivo de avaliar a sua evolução, interação com minerais e rochas e interconexões do reservatório em subsuperfície. As amostras são ricas em material dissolvido, com condutividade elétrica variando de $84 \mathrm{mS} / \mathrm{cm}$ a $137 \mathrm{mS} / \mathrm{cm}$ aumentando com a profundidade de extração. Também foi observado um enriquecimento em isótopos pesados de $\mathrm{H}$ e $\mathrm{O}$ em direção aos níveis mais profundos, possivelmente causado pela interação água-rocha, potencializada pela elevada temperatura, característica dos níveis mais profundos, e pelo longo período de residência dessas águas. À profundidade de 1450 a 1520 metros, zona de produção de 7 dos 10 poços amostrados, verifica-se uma pequena variação da composição isotópica e da CE, indicando uma homogeneidade das amostras nesse nível do reservatório. No nível de produção mais raso, situado a 450 metros abaixo da superfície, obteve-se os menores valores de $\delta^{2} \mathrm{H}, \delta^{18} 0$ e de condutividade elétrica, com composição isotópica similar a da água do mar, possivelmente representando a água que entrou na formação durante a sedimentação da bacia.

Palavras-chave: geofísica isotópica, água de formação, isótopos estáveis, Bacia do Recôncavo, destilação criogênica.

\footnotetext{
${ }_{1}$ Instituto de Física e Centro de Pesquisa em Geofísica e Geologia, Universidade Federal da Bahia, Salvador, BA, Brasil. Tels.: +55(71) 3283-6680/ 3283-6691 -E-mails: danilosateles@yahoo.com.br; expedito@ufba.br

2 Instituto Federal Baiano, Salvador, BA, Brasil-E-mail: cplsgs@gmail.com
} 


\section{INTRODUÇÃo}

Os isótopos estáveis ambientais vêm cada vez mais sendo utilizados em estudos hidrogeológicos na investigação de problemas hidrológicos tais como identificação de mecanismos de salinização em águas subterrâneas, definição de áreas de recarga, quantificação e origem de mistura entre unidades hídricas. Aplicado à água de formação, resíduo ligado à atividade de extração do petróleo, o estudo desses isótopos permite levantar hipóteses a respeito do grau de evolução hidrológica do campo, da sua classificação hidroquímica e principalmente da classificação genética, ou seja, sua origem. A partir desses resultados é possível determinar se o reservatório, que armazena esta água, tem ligação com outros reservatórios, aquíferos ou se sofre infiltração de água meteórica. Worden et al. (2006) analisando os isótopos do oxigênio, hidrogênio, carbono e estrôncio e a salinidade das águas de formação da Formação Chaunoy na Bacia de Paris concluíram haver, nesse local, uma dependência da salinidade da água com a profundidade em que a mesma se encontra e com a localização geográfica dos poços, sendo que a concentração de solutos totais dissolvidos decresce do norte para o sul da bacia devido à entrada de água doce principalmente de origem meteórica. Segundo os autores, os isótopos analisados apresentaram valores bem similares aos das águas meteóricas atuais, 0 que sugere que a entrada dessas águas é um fenômeno recente e se dá devido a falhas localizadas principalmente na faixa aflorante da formação sul da bacia. As altas concentrações de solutos, encontradas no nível mais profundo dos poços localizados no norte da bacia, foram interpretadas como originadas, principalmente, da interação água-rocha a partir da dissolução de halita e que se moveram em um determinado período, interagindo intensamente com as rochas, levando a um enriquecimento considerável no $\delta^{18} 0$. A parte central da bacia ainda conserva a água aprisionada durante a sua formação (água conata), que não interagiu tão intensamente com as rochas. Estas águas, provavelmente de origem paleometeórica, moveram-se por convecção para a porção leste da bacia devido ao gradiente de temperatura em diferentes pontos, levando ao fracionamento do líquido, enriquecendo-0 em isótopos pesados.

A partir do estudo de traçadores como $0 \mathrm{Cl}, \mathrm{Br}, \delta^{2} \mathrm{H}$ e $\delta^{18} 0$ e da salinidade, Ziegler et al. (2001) descrevem um modelo paleo-hidrodinâmico para a origem e movimentação de fluidos no Campo de Oseberg (Mar do Norte), concluindo que houve a entrada de água de origem marinha, após a principal fase de migração do óleo. Isto foi indicado pela diferença da composição isotópica da água deste poço em relação aos outros, o que evidenciou uma mistura de água do mar e água meteórica atual.
Rostron \& Holmden (2003) analisaram a razão isotópica do oxigênio em águas de formação amostradas em campos da Bacia de Williston (Canadá - EUA), concluindo que as mesmas sofrem infiltrações de água em determinadas áreas, levando a um empobrecimento em isótopos pesados em seus aquíferos. Além disso, identificaram um lento fluxo de "salmoura" em direção a áreas estagnadas, bem como a presença de água glacial recente que indica uma recarga em subsuperfície.

De acordo com Morad et al. (2003) as águas de formação podem ser originadas a partir das águas conatas, águas oceânicas, águas oceânicas evaporadas, águas meteóricas, águas subterrâneas evaporadas ou ainda, águas magmáticas. Ainda segundo os autores, quase toda a água de formação sofre interações com rochas resultando em uma evolução de sua composição isotópica.

A contaminação do poço, com águas ricas em oxigênio, ou não, nutrientes e microorganismos, como as águas meteóricas, pode causar a biodegradação do óleo, acarretando perda de suas frações nobres, diminuindo o seu grau API, elevando a sua viscosidade e acidez, tornando dispendiosos a produção, transporte, processamento e refino (Seewald, 2003).

Neste trabalho foi feita a análise isotópica e medido o pH e a condutividade de águas de formação de um campo de petróleo e gás da Bacia do Recôncavo, em poços livres de recuperação secundária procurando determinar, a partir desses resultados, os parâmetros que controlam essa composição, tais como contato com água superficial, interação água-rocha, contato entre poços. Além disso, foram analisados métodos para tratamento e análise de águas hipersalinas.

\section{TRATAMENTO DE AMOSTRAS E AMOSTRAGEM}

A preparação e análise das amostras foram executadas no Laboratório de Física Nuclear Aplicada, LFNA/UFBA, sendo elas provenientes de um campo da Bacia do Recôncavo e cedidas pela Petrobras. Durante o processo de amostragem houve bastante cautela nas etapas de transporte e armazenamento, evitando-se perdas através da evaporação e/ou trocas isotópicas com o meio. Por isso, visando assegurar a pureza das amostras coletadas, bem como a padronização dos métodos de amostragem, a metodologia adotada nas coletas e na estocagem das amostras procurou seguir o protocolo indicado por Lemay (2002). As amostras foram retiradas na cabeça do poço, e são constituídas basicamente de uma mistura de água, óleo e gás. Elas foram inicialmente armazenadas em um "barrilete" de 5 litros, lacradas e deixadas em repouso por 24 horas para que decantassem e, dessa forma, sepa- 
rassem o óleo da água e então, por diferença de densidade, a água ocupasse a parte inferior do recipiente fazendo o óleo flutuar. Posteriormente, a água foi retirada por uma torneira disposta na parte inferior do recipiente, filtrada em lã de vidro e em um filtro qualitativo passando em seguida por um novo processo de filtração, agora utilizando um filtro Millipore ${ }^{\circledR}$, que retém partículas de até $0,45 \mu \mathrm{m}$. A água foi então armazenada, em frascos de vidro de 1 litro e frascos menores de $100 \mathrm{ml}$ e fechados com batoque e tampas de plástico e acondicionada em geladeira. Inicialmente, as amostras de oxigênio foram preparadas em uma linha de extração, separada do espectrômetro, a partir da técnica de micro-equilíbrio da água com $\mathrm{CO}_{2}$, e ficavam em repouso em um banho ultratermostático, a $25^{\circ} \mathrm{C}$ por 24 horas para em seguida, a partir da linha de extração, captura-se $0 \mathrm{CO}_{2}$ resultante que, equilibrado com a água, revelava a sua composição isotópica. Com o processo de modernização do laboratório, um novo equipamento foi introduzido, o GasBench II da ThermoFinnigan, ligado diretamente ao espectrômetro de massas, que opera utilizando fluxo contínuo. Para várias amostras foram obtidos resultados usando os dois métodos, sendo que os seus resultados foram comparados, o que serviu como uma certificação do seu bom funcionamento além da sua precisão e reprodutibilidade.

\section{Análise isotópica do hidrogênio e do oxigênio de água hipersalina}

Para determinar a razão Deutério/Hidrogênio $\left({ }^{2} \mathrm{H} /{ }^{1} \mathrm{H}\right)$ é utilizado, principalmente, o método apresentado por Brand et al. (2000), que consiste em obter o hidrogênio que constitui a água, através da redução da mesma a $850^{\circ} \mathrm{C}$ com cromo metálico, em um aparelho denominado H-Device que é diretamente ligado ao espectrômetro de massa.

Alíquotas de aproximadamente 2,0 $\mu$ l da amostra são injetadas nesse reator, ilustrado na Figura 1, com consequente liberação do $\mathrm{H}_{2}$ representado pela equação abaixo:

$$
3 \mathrm{H}_{2} \mathrm{O}+2 \mathrm{Cr} \rightarrow \mathrm{Cr}_{2} \mathrm{O}_{3}+3 \mathrm{H}_{2}
$$

0 hidrogênio é então direcionado para o espectrômetro de massa onde é analisado em relação a um gás de referência. Quando se trata de águas hipersalinas, como geralmente são as águas de formação, o processo de oxi-redução é comprometido devido à grande quantidade de sais totais dissolvidos que precipita e danifica 0 reator. Como alternativa para este problema há vários trabalhos na literatura especializada, dentre os quais Bourg et al. (2001) que utilizaram o método de equilíbrio água-gás, e que chegaram à conclusão de que havia um certo efeito da concentração dos solutos sobre a razão $\mathrm{D} / \mathrm{H}$ da amostra em relação à razão
${ }^{2} \mathrm{H} /{ }^{1} \mathrm{H}$ da água pura, $\mathrm{O}$ que tornaria o processo inviável. Em contrapartida, concluíram não haver significante influência da salinidade nos isótopos do oxigênio. Koehler et al. (1991) realizaram medidas da composição isotópica de soluções salinas com diferentes concentrações utilizando destilação por micro-equilíbrio, com o qual chegou a conclusão de não haver variação isotópica da amostra com o aumento da quantidade de sais totais dissolvidos, no que diz respeito ao hidrogênio, porém notou um certo fracionamento que ocorre devido aos sais de Mg contidos na solução analisada. Nessas águas um pré-tratamento faz-se necessário, visto que, 0 excesso de sais totais dissolvidos seria precipitado durante 0 processo de oxi-redução, danificando o reator que contém 0 cromo metálico. 0 pré-tratamento consiste em destilar a amostra de modo a retirar os sais totais dissolvidos, mas preservando integralmente suas características isotópicas, possibilitando desta forma, a análise em espectrômetro de massa a partir do H-Device.

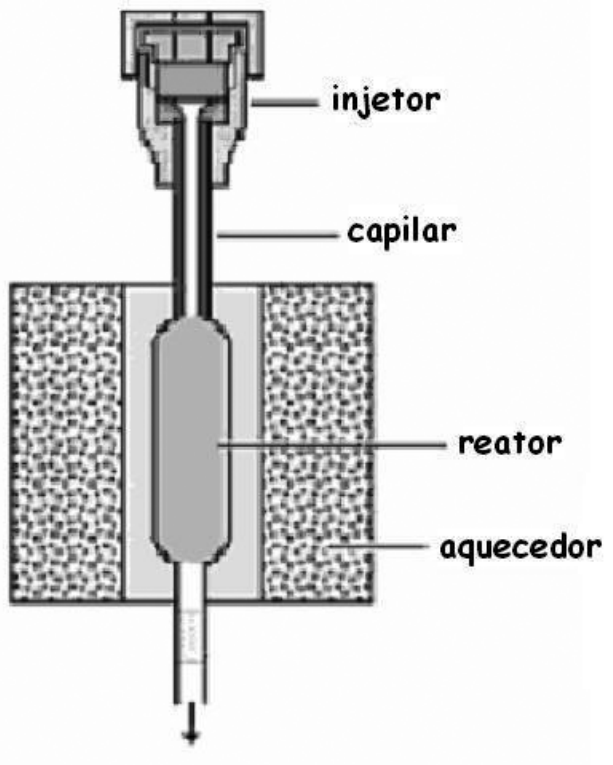

\section{Saída para o IRMS}

Figura 1 - Reator de oxi-redução contendo cromo-metálico para análise isotópica do hidrogênio da água. Fonte: Groot (2008).

\section{Processo de destilação da água hipersalina}

A extração dos sólidos dissolvidos foi obtida a partir de uma destilação criogênica sob vácuo. 0 aparato de destilação consiste de uma linha cilíndrica de aproximadamente $150 \mathrm{~cm}$ ligada lateralmente a uma bomba evacuadora e inferiormente a seis linhas mais curtas de aproximadamente $25 \mathrm{~cm}$ de comprimento, independentes uma da outra, e tendo encaixados em suas extremidades dois balões de volume $100 \mathrm{ml}$ cada como ilustra a Figura 2. 


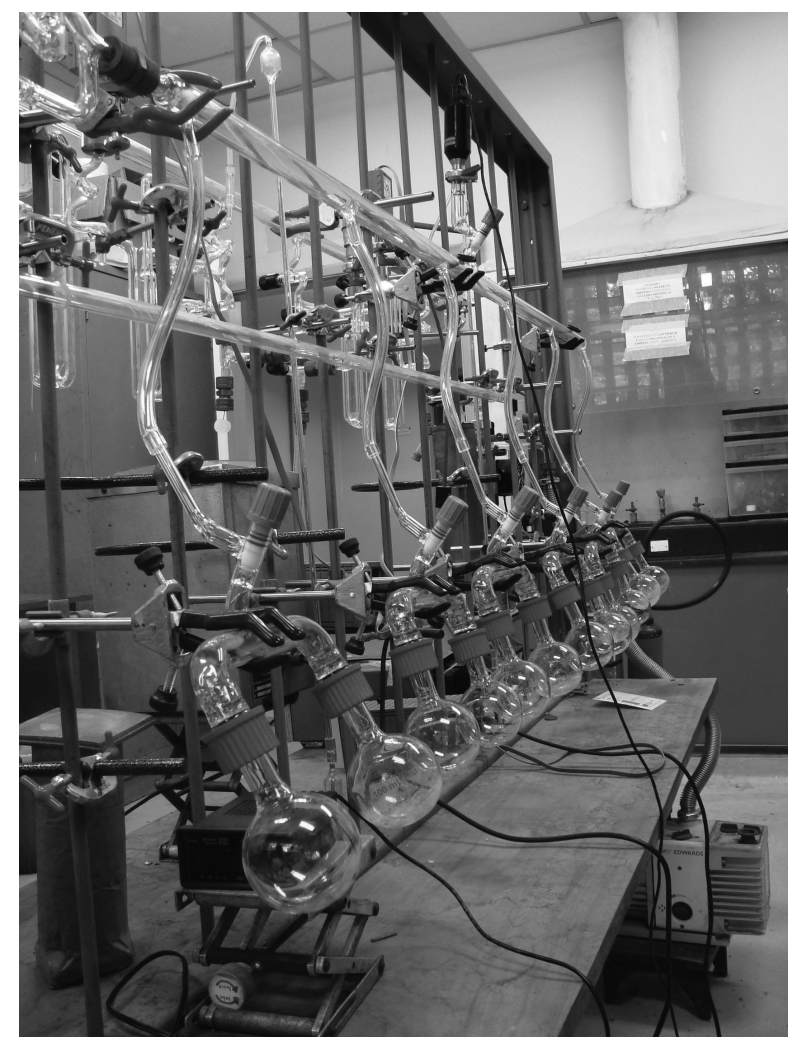

Figura 2 - Aparato utilizado para a destilação criogênica da água de formação hipersalina.

Após passar pelo processo de filtração, aproximadamente $15 \mathrm{ml}$ da amostra foram colocados em um dos balões (balão amostrador). Em seguida o mesmo foi mergulhado em nitrogênio líquido por aproximadamente 10 minutos, tempo suficiente para que toda a amostra congelasse. Posteriormente, evacuou-se cada par isoladamente, Figura 3, de modo a evitar a contaminação entre as amostras, obtendo-se um ambiente de baixa pressão $\left(<10^{-2}\right.$ mbar). Obtendo-se a pressão desejada, 0 nitrogênio líquido foi retirado e 0 balão, com a amostra, aquecido em banhomaria a cerca de $40^{\circ} \mathrm{C}$. A água evapora e condensa no outro balão, mergulhado em nitrogênio líquido, denominado de balão coletor. Observada a evaporação quase completa da água, após um tempo médio de aproximadamente 20 minutos, 0 balão amostrador é aquecido a $150^{\circ} \mathrm{C}$ com uma manta térmica por aproximadamente 5 minutos a fim de se obter a evaporação completa da água da amostra e capturá-la no balão coletor de modo a evitar 0 seu fracionamento isotópico. No balão coletor toda a água coletada congelada, se funde sob temperatura ambiente, e em seguida é recolhida num frasco de vidro de $15 \mathrm{ml}$ fechado com batoque e tampa de plástico. Devido ao volume de sal precipitado no balão amostrador, observa-se uma redução do volume de água coletada em comparação com a amostrada.

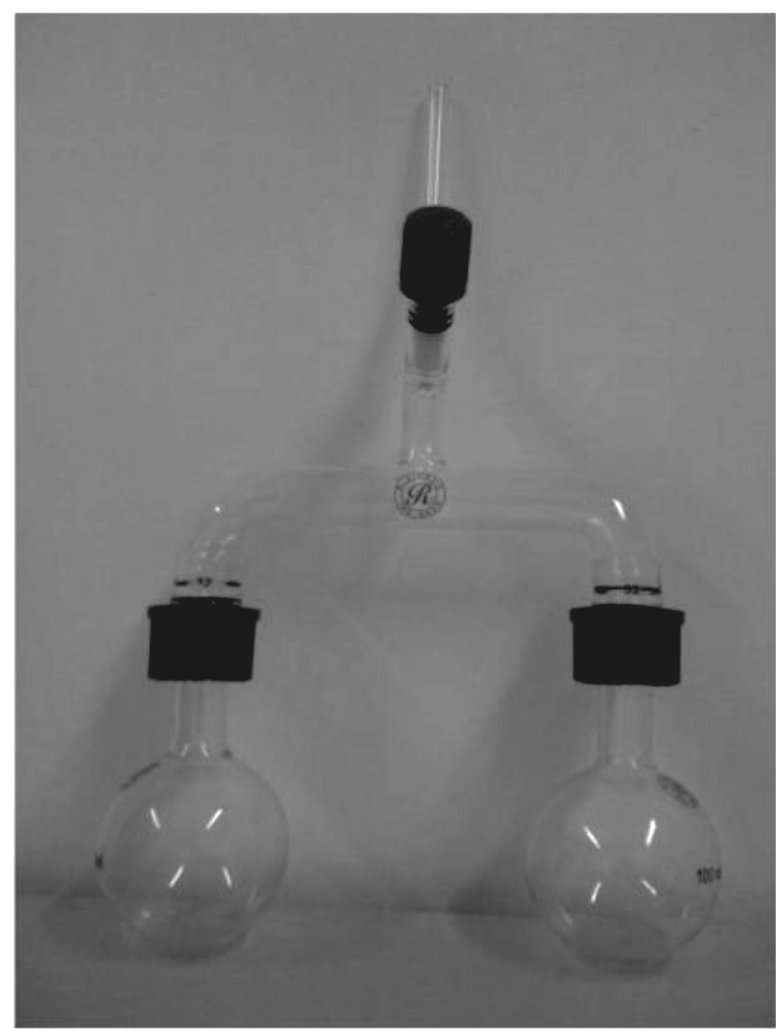

Figura 3 - Esquema detalhado dos frascos coletor e amostrador da linha de extração. Fonte: West et al. (2006).

Na destilação deve-se evitar o fracionamento isotópico que é uma variação na razão isotópica de um determinado elemento. Ressalta-se que na natureza são justamente variações na abundância isotópica de elementos tais como H, C, N, O, Si e S que tornam possível sua utilização como traçadores. Em laboratório, depois de coletada uma determinada amostra, o fracionamento deve ser evitado ao máximo, ou do contrário, levaria a uma perda da identidade isotópica da mesma descaracterizandoa. Como o método de extração do sal poderia causar o fracionamento da amostra, algumas precauções tiveram que ser tomadas a fim de garantir que a sua razão isotópica não variasse. A eficiência do método de destilação e a reprodutibilidade das medidas foram aferidas através da destilação de águas sintéticas, que foram preparadas misturando-se quantidades pré-determinadas de sal marinho em uma água matriz, cuja composição isotópica era conhecida. Ao fim da destilação dessas águas elas foram analisadas e as suas razões isotópicas comparadas com os valores isotópicos da água matriz, devendo essa diferença estar dentro de um desvio avaliado de $\pm 1 \%$ para o hidrogênio e de $\pm 0,1 \%$ no que diz respeito ao oxigênio.

Na Tabela 1 apresentam-se as características de salinidade das amostras sintéticas bem como sua composição isotópica e 
Tabela 1 - Análise isotópica das águas sinteticamente salinizadas e sua matriz.

\begin{tabular}{|c|c|c|c|c|c|c|c|c|c|c|c|c|c|}
\hline \multicolumn{14}{|c|}{ Amostras de águas sintéticas } \\
\hline \multirow{2}{*}{ Amostra } & \multicolumn{5}{|c|}{$\delta \mathrm{D}(\%)$} & \multicolumn{4}{|c|}{$\delta^{18} 0(\% 0) \mathrm{s} /$ dest. } & \multicolumn{4}{|c|}{$\delta^{18} 0(\%) c /$ dest. } \\
\hline & M1 & M2 & M3 & M4 & Média & M1 & M2 & M3 & Média & M1 & M2 & M3 & Média \\
\hline matriz & 0,8 & 0,3 & $N A^{*}$ & $N A^{*}$ & 0,6 & $-1,1$ & $-1,3$ & $-1,0$ & $-1,1$ & $-1,3$ & $-1,2$ & $N A^{*}$ & $-1,3$ \\
\hline matriz dest. & 0,8 & 0,4 & 1,0 & 1,0 & 0,8 & $-1,3$ & $-1,2$ & NA & $-1,2$ & $-1,3$ & $-1,2$ & $N A^{*}$ & $-1,2$ \\
\hline $60 \mathrm{~g} / \mathrm{l}$ & $-0,1$ & 0,7 & 0,6 & $N A^{*}$ & 0,4 & $-1,2$ & $-1,1$ & $N A^{*}$ & $-1,2$ & $-1,2$ & $-1,3$ & $N A^{*}$ & $-1,2$ \\
\hline $120 \mathrm{~g} / \mathrm{l}$ & $-0,6$ & $-1,4$ & $-0,5$ & 0,4 & $-0,5$ & $-1,4$ & $-1,3$ & $-1,1$ & $-1,3$ & $-1,1$ & $-1,2$ & $-1,0$ & $-1,1$ \\
\hline $240 \mathrm{~g} / \mathrm{l}$ & $-1,2$ & $-1,3$ & $-1,5$ & $-1,5$ & $-1,4$ & $-1,2$ & $-1,4$ & $-1,3$ & $-1,3$ & $-1,2$ & $-1,4$ & $-1,1$ & $-1,2$ \\
\hline
\end{tabular}

*Não analisado.

seu desvio com relação à água matriz, cuja composição isotópica, com e sem destilação, também é apresentada. Os resultados das análises dessas águas apresentam um $\delta \mathrm{D}$ médio de $1 \%$ e $\delta^{18} 0$ médio de $-1,2 \%$, ambos calculados em relação ao padrão internacional VSMOW, com desvios dentro de $1 \%$ para $0 \delta \mathrm{D}$ e $0,1 \%$ para $0 \delta^{18} 0$. A composição isotópica após a destilação não sofreu grandes variações entre si e em relação à água matriz atestando a confiabilidade e reprodutibilidade do método de destilação criogênica.

\section{ÁREA DE ESTUDO}

A Bacia do Recôncavo tem aproximadamente 11500 km² de área. Localizada no Estado da Bahia, foi a primeira bacia petrolífera explorada no Brasil, possuindo hoje mais de 5000 poços perfurados. Sua origem e evolução estão ligados à formação do Atlântico Sul e da Margem Continental Brasileira, guardando um importante registro geológico na forma de uma seção lacustre que inclui basicamente folhelhos e arenitos. Segundo Bruhn \& De Ros (1987) a coluna estratigráfica do Recôncavo, ilustrada na Figura 4 , divide a bacia, da base para o topo, nas formações Aliança, Sergi, Itaparica, Candeias, Salvador, Marfim, Pojuca, São Sebastião e Marizal. Suas principais rochas geradoras de petróleo estão incluídas na Formação Candeias, sendo representadas por folheIhos escuros e cinza-esverdeados que ocorrem intercalados com folhelhos calcíferos pretos, lentes micríticas de calcários e turbiditos silicicláticos (Milhomem et al., 2003).

Segundo Gaglianone \& Trindade (1988) dados geoquímicos caracterizam o paleoambiente deposicional das rochas geradoras como lacustre de água doce com fundo anóxico, o que propiciou a eficiência da preservação da matéria orgânica ali depositada, sendo esta predominantemente rica em lipídios, derivada principalmente de algas, bactérias e alguns vegetais superiores (angiospermas).

Para este trabalho, extraiu-se um total de 12 amostras em 10 poços diferentes, situados em profundidades que variaram de 450 metros a 1800 metros, drenando 4 níveis diferentes de produção desse campo, cuja seção geológica é apresentada na Figura 5. Trata-se de um campo maduro de produção, com grande porcentagem de água nos fluidos extraídos.

Segundo Coste \& Valois (2000), campos maduros são aqueles que possuem um histórico de produção relativamente longo, com muitos poços perfurados e que atualmente apresentam declínio na produção e que necessitam de operação e tecnologias específicas para recuperar a sua rentabilidade como é 0 caso da referida bacia. Espera-se que a análise da composição isotópica da água de formação possa contribuir para o conhecimento da dinâmica dos fluidos no reservatório e aumentar o fator de recuperação desses campos.

\section{RESULTADOS}

Na Tabela 2 apresentam-se o pH, a condutividade elétrica, profundidade média de cada nível de produção bem como o resultado da composição isotópica das amostras. $0 \mathrm{pH}$ das águas coletadas, exceto pela amostra do poço 10_2, variou entre 5,9 e 7,5 evidenciando um caráter neutro a ligeiramente ácido. A amostra 10_2, que representa uma segunda coleta no poço 10, possivelmente teve 0 seu pH alterado por algum fator externo, que embora tenha alterado o seu valor, não comprometeu a composição isotópica da amostra. Os resultados do $\delta^{18} 0$ apresentados foram obtidos, a partir dos métodos por equilíbrio "off-line", com introdução via dual-inlet, e por fluxo contínuo usando o GasBench ${ }^{\circledR}$. Para as medidas de $\delta$ D são apresentados os resultados feitos por injeção manual no H-Device e por injeção através de amostrador automático.

Para garantir a eficiência de cada destilação, a razão isotópica ${ }^{18} 0 /{ }^{16} 0$ foi medida nas amostras destiladas e não destiladas, sendo posteriormente comparadas verificando-se não haver, dentro do desvio aceitável, variações significativas na composição 


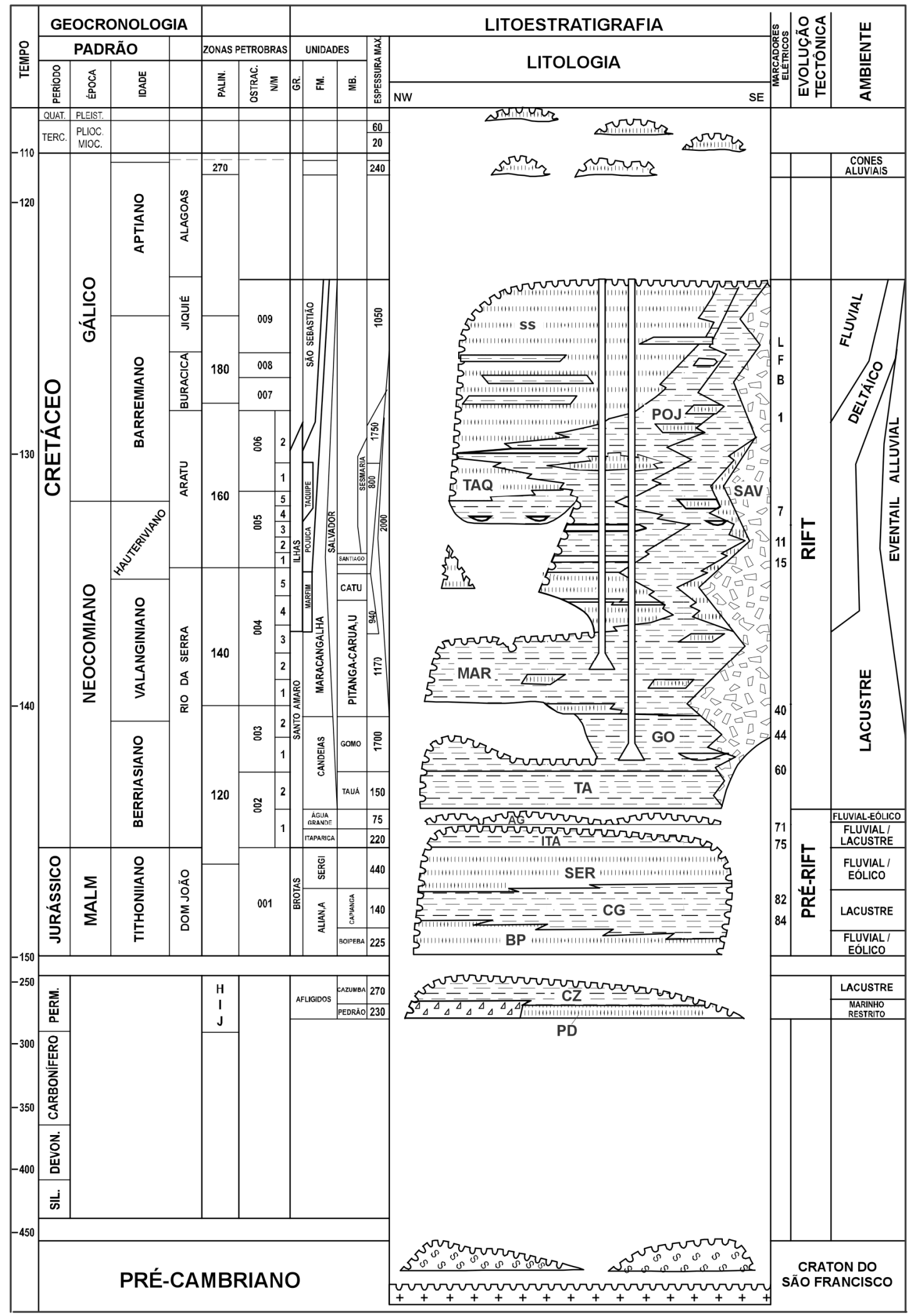

Figura 4 - Coluna estratigráfica da Bacia do Recôncavo (Caixeta et al., 1994). 


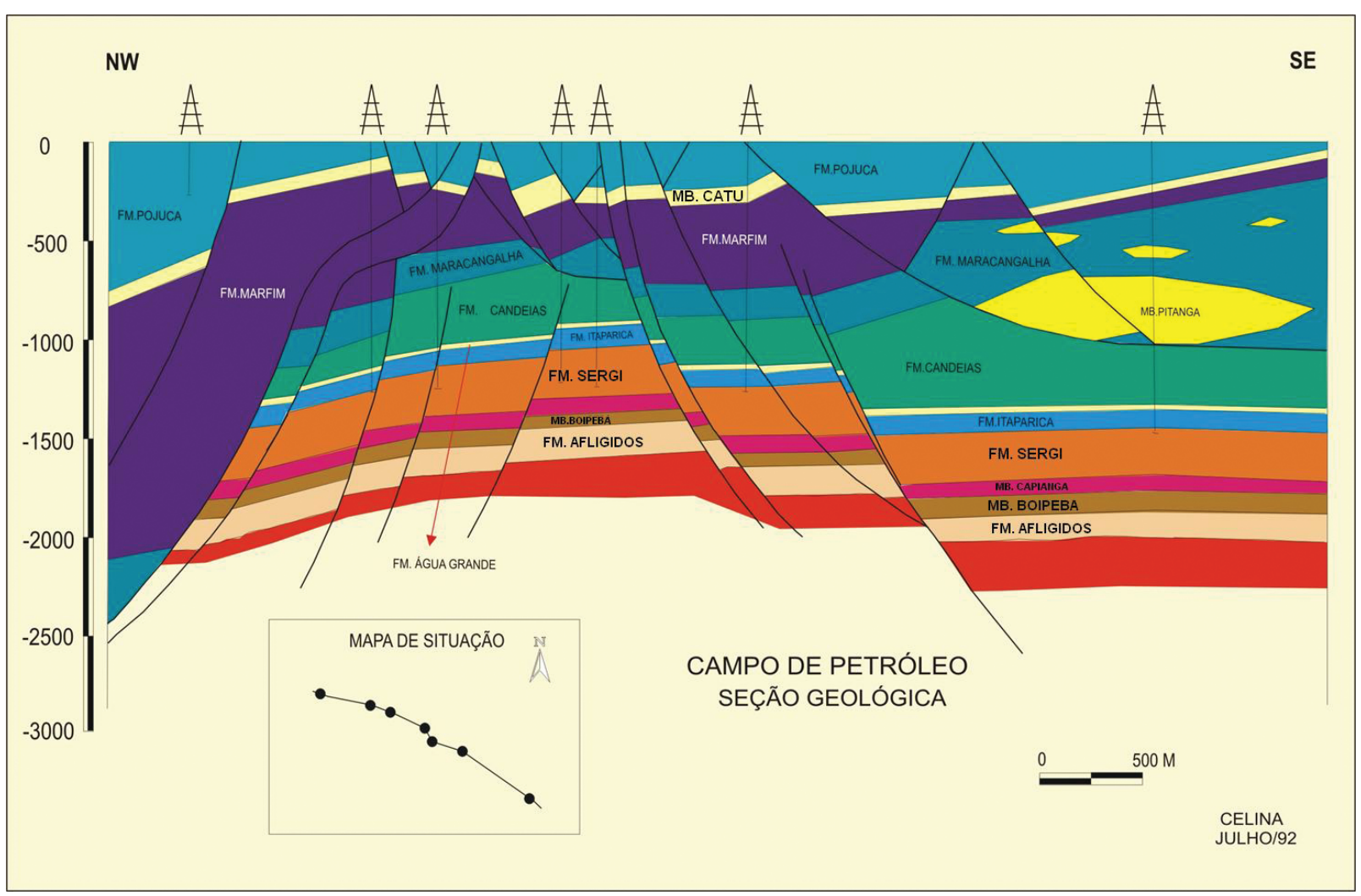

Figura 5 - Seção geológica de um campo de petróleo da Bacia do Recôncavo.

isotópica do oxigênio devido à destilação. Nota-se, porém, um pequeno enriquecimento em ${ }^{18} 0$ das amostras destiladas em relação às não destiladas, onde supõe-se ser resultado da hipersalinidade das águas que influenciaria o tempo de equilíbrio água - gás, no GasBench.

\section{DISCUSSÃO}

\section{Salinidade}

Segundo Archer \& Wall (1994), a salinidade das águas de formação é a principal forma de controle da sua resistividade e um parâmetro essencial para o cálculo de reservas de óleo. Na água, a condutividade é diretamente relacionada com o seu conteúdo iônico. A concentração de sólidos totais dissolvidos (STD) é um índice de quantidade de substâncias dissolvidas na água e é diretamente proporcional à condutividade elétrica. Nelas a CE e consequentemente 0 teor de STD são bastante variáveis, variando desde essencialmente doces com menos de 0,9 $\mu \mathrm{S} / \mathrm{cm}(1,0 \mathrm{~g} / \mathrm{l})$ até salmouras com mais de 248,7 mS/cm, (250,0 g/l) (Levorsen, 1958). Nos reservatórios analisados, os valores da condutividade elétrica das amostras variaram de 84 a $137 \mathrm{mS} / \mathrm{cm}$, que correspondem aproximadamente a uma faixa de valores de STD numericamente equivalente de 84 a $137 \mathrm{~g} / \mathrm{l}$. Os valores mais baixos de 84 e $86 \mathrm{mS} / \mathrm{cm}$ foram registrados na zona de produção de 435 a 486 metros, e com valores variando na faixa de 120 a $140 \mathrm{mS} / \mathrm{cm}$ nos outros níveis de produção. Tal variabilidade evidencia uma interação da água, em níveis mais profundos com rochas, dissolvendo seus minerais. Tal efeito é potencializado pelo longo tempo de armazenamento dessas águas e pelas altas temperaturas características de maiores profundidades.

\section{Composição isotópica}

Os resultados obtidos, mostrados na Tabela 2, apresentam uma composição isotópica que varia entre $\approx 2 \%$ a $\approx 16 \%$ para 0 $\delta \mathrm{D}$ e entre $\approx 0,1 \%$ e $\approx 3,4 \%$ para $\delta^{18} 0$. Relacionando a composição isotópica com 0 nível de extração (Figs. 6 e 7) percebe-se, analogamente à condutividade elétrica, que 0 enriquecimento se dá em direção aos níveis mais profundos de extração, homogeneizando-se em grandes profundidades, como ilustra as referidas figuras.

Das 12 amostras analisadas quase todas, com exceção da produzida no nível mais raso apresentaram uma composição 
Tabela 2 - pH, condutividade e dados isotópicos da água de formação de poços amostrados.

\begin{tabular}{|c|c|c|c|c|c|c|c|c|c|}
\hline \multirow{2}{*}{ Amostra } & \multirow{2}{*}{$\begin{array}{l}\text { Profundidade } \\
\text { (m) }\end{array}$} & \multirow{2}{*}{$\mathrm{pH}$} & \multirow{2}{*}{ CE } & \multicolumn{2}{|c|}{$\begin{array}{c}\delta^{18} 0(\%) \\
\text { (sem destilar) }\end{array}$} & \multicolumn{2}{|c|}{$\begin{array}{l}\delta^{18} 0(\% 0) \\
\text { (destilado) }\end{array}$} & \multicolumn{2}{|c|}{$\delta^{2} \mathrm{H}(\% 0)$} \\
\hline & & & & $\begin{array}{l}\text { Dual } \\
\text { Inlet }\end{array}$ & $\begin{array}{c}\text { Gas } \\
\text { Bench }\end{array}$ & $\begin{array}{l}\text { Dual } \\
\text { Inlet }\end{array}$ & $\begin{array}{c}\text { Gas } \\
\text { Bench }\end{array}$ & $\begin{array}{l}\text { Dual } \\
\text { Inlet }\end{array}$ & $\begin{array}{c}\text { Auto } \\
\text { Sampler }\end{array}$ \\
\hline Poço 1_1 & $435,0-486,0$ & 7,4 & 86,3 & $-0,1$ & $-0,1$ & $-0,1$ & $-0,2$ & 2,0 & 3,3 \\
\hline Poço 1_2 & $435,0-486,0$ & 7,5 & 84,0 & $N A^{*}$ & $-0,1$ & $N A^{*}$ & $-0,1$ & $N A^{*}$ & 3,1 \\
\hline Poço 2 & $1266,5-1268,5$ & 6,7 & 127,5 & 1,5 & 1,4 & 3,4 & 1,5 & 9,4 & 9,4 \\
\hline Poço 3 & $1456,5-1458,0$ & 6,5 & 133,5 & 3,0 & 3,0 & 3,0 & 3,0 & 14,8 & 13,9 \\
\hline Poço 4_1 & $1443,0-1457,5$ & 6,1 & 130,2 & 2,8 & 2,7 & 2,9 & 3,0 & 13,9 & 13,6 \\
\hline Poço 4_2 & $1443,0-1457,5$ & 6,0 & 127,4 & $N A^{*}$ & 2,5 & $N A^{*}$ & 3,0 & $N A^{*}$ & 14,8 \\
\hline Poço 5 & $1441,5-1479,0$ & 6,6 & 131,9 & 3,1 & 2,4 & 2,9 & 2,9 & 13,9 & 14,1 \\
\hline Poço 6 & $1485,0-1508,5$ & 6,2 & 137,2 & $N A^{*}$ & 2,8 & $N A^{*}$ & 2,8 & $N A^{*}$ & 13,6 \\
\hline Poço 7 & $1498,0-1541,5$ & 6,5 & 127,4 & $N A^{*}$ & 2,6 & $N A^{*}$ & 2,6 & $N A^{*}$ & 12,7 \\
\hline Poço 8 & $1454,0-1500,0$ & 6,7 & 135,3 & 3,7 & 3,1 & 3,4 & 3,4 & 15,6 & 15,5 \\
\hline Poço 9 & $1448,0-1451,0$ & 5,9 & 126,6 & $N A^{*}$ & 2,6 & $N A^{*}$ & 2,7 & $N A^{*}$ & 14,5 \\
\hline Poço 10_1 & $1805,0-1815,5$ & 5,9 & 116,7 & 2,8 & 2,4 & 2,7 & 2,8 & 14,0 & 14,4 \\
\hline Poço 10_2 & $1805,0-1815,5$ & 4,4 & 131,8 & $N A^{*}$ & 2,5 & $N A^{*}$ & 2,7 & $N A^{*}$ & 13,2 \\
\hline
\end{tabular}

*Não analisado.

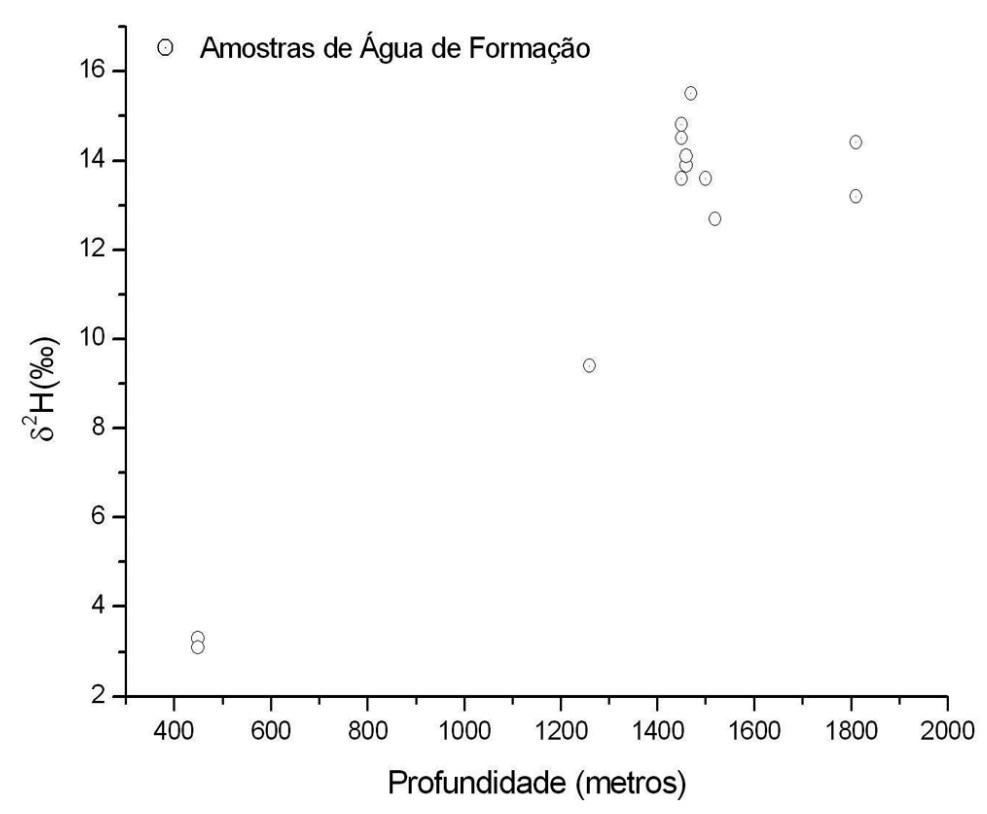

Figura 6 - Relação $\delta^{2} \mathrm{H}$ versus profundidade de águas de formação da Bacia do Recôncavo.

isotópica rica em ${ }^{2} \mathrm{H}$ e ${ }^{18} 0 \mathrm{em}$ relação à água do mar. Construindo o diagrama $\delta \mathrm{D}$ Vs $\delta^{18} 0$, ilustrado na Figura 8, verifica-se que a distribuição dos pontos se alinha em torno da reta de regressão linear com o coeficiente angular bem menor que a linha meteórica global e bem mais enriquecida que a água do mar.

A amostra com composição isotópica próxima à linha meteórica global está localizada no nível mais raso da formação, a uma profundidade de 300 metros, possivelmente represente 


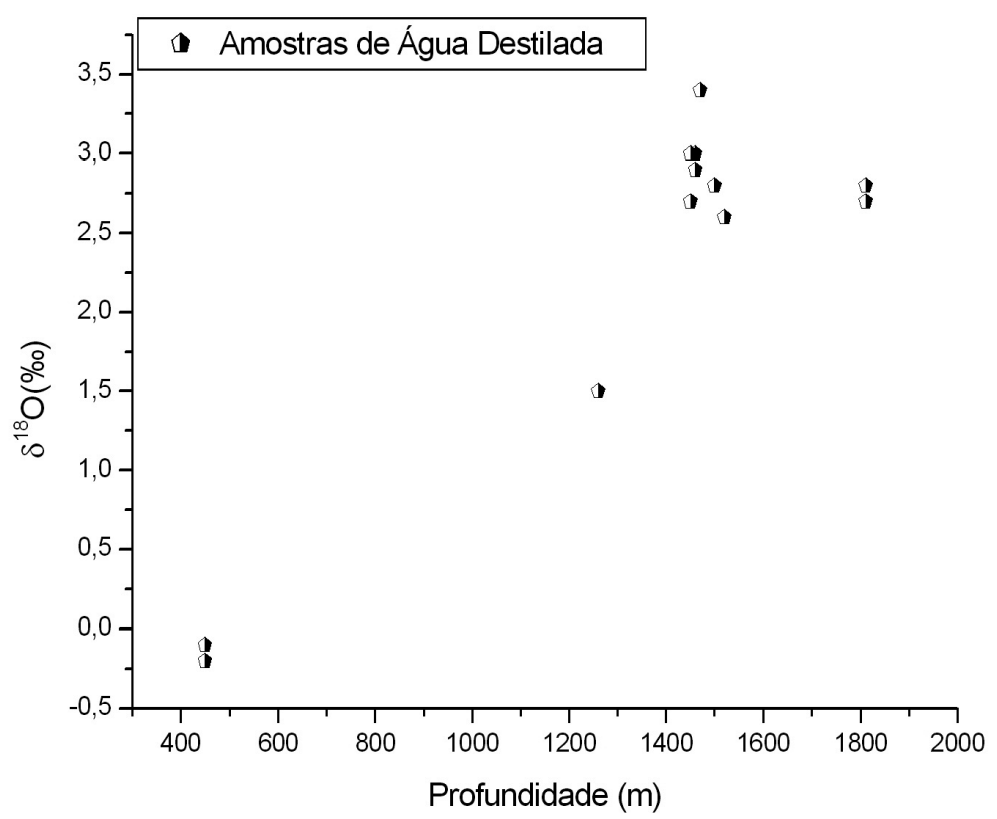

Figura 7 - Relação $\delta^{18} 0$ versus profundidade de águas de formação da Bacia do Recôncavo.

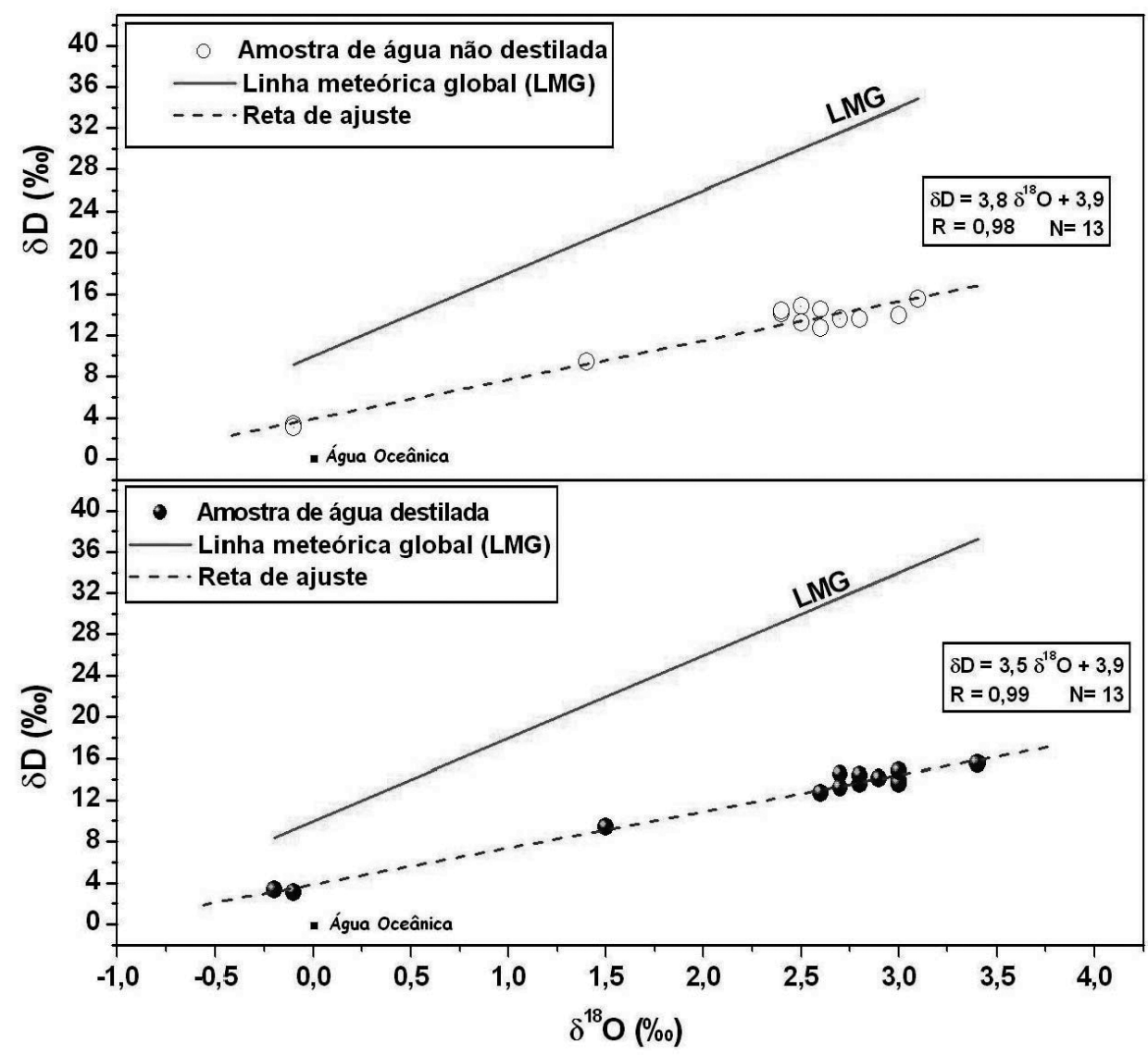

Figura 8 - Diagrama $\delta^{2} \mathrm{H}$ versus $\delta^{18} 0$ das amostras de águas de formação, destiladas e não destiladas, da Bacia do Recôncavo. 
a composição isotópica da água que entrou na bacia durante a sua deposição. Porém, como o poço em que estas amostras foram retiradas está situado próximo a uma zona de recuperação secundária, feita a partir da injeção de água, pode ter havido uma eventual contaminação das mesmas fazendo variar a sua composição isotópica. A hipótese de uma possível infiltração de água meteórica recente é descartada pois, segundo Gaglianone \& Trindade (1988), não há evidencias de biodegradação nos óleos da Bacia do Recôncavo.

A composição isotópica das amostras provenientes de profundidades entre 1450 e 1800 metros estão distribuídas numa faixa muito estreita sugerindo não haver compartimentabilização nessa zona de produção.

Os resultados plotados no diagrama $\delta^{2} \mathrm{H} \times \delta^{18} 0$ se alinham ao longo de uma linha de regressão linear com coeficiente angular menor que 0 da linha meteórica global, provavelmente resultado da maior interação água-rocha com 0 aumento da profundidade. A depleção em ${ }^{2} \mathrm{H} \mathrm{e}{ }^{18} 0$ poderia estar relacionada a processos de filtragem natural da água através de folhelhos e argilas compactados que agiriam como membranas semipermeáveis (Coplen \& Hanshaw, 1973).

\section{CONCLUSÕES}

A aplicação da técnica de destilação criogênica de águas hipersalinas permitiu a análise isotópica da água de formação de um campo de petróleo da Bacia do Recôncavo, cujo teor de sais totais dissolvidos era muito superior a da água do mar, possibilitando, juntamente com a análise físico-químico, a identificação da dinâmica do fluxo subterrâneo daquele líquido bem como a interação com sua vizinhança. A destilação criogênica de águas hipersalinas revelou-se segura e reprodutível à análise isotópica, não interferindo significativamente na composição isotópica das amostras, isto é, não gerou o fracionamento e consequentemente a perda da identidade isotópica da mesma, podendo, portanto ser aplicável, não só a amostras de águas de formação como também a qualquer solução aquosa que contenha uma alta concentração de solutos dissolvidos.

Os gráficos de $\delta \mathrm{D}$ e $\delta^{18} 0$ versus a profundidade, assim como a análise da condutividade elétrica, indicam um enriquecimento em isótopos pesados e em solutos dissolvidos com a profundidade 0 que sugere uma forte interação água-rocha, potencializada pelo movimento e pelo longo período de armazenamento da água, em níveis mais profundos do campo. 0 poço 1 , localizado no nível mais raso, apresentou uma composição isotópica próxima a da água do mar. Tal resultado sugere que essa amostra contém a composição isotópica da água original que entrou na formação durante a sedimentação da bacia, desde que a falta de correlação com água meteórica superficial é atestada pela inexistência de biodegradação dos óleos deste campo.

Os valores de $\delta \mathrm{D}$ e $\delta^{18} 0$ apresentam uma boa correlação, e se alinham na regressão linear com um coeficiente angular menor que o da linha meteórica global, estando os dados isotópicos fora e à direita dessa mesma linha, o que reforça a hipótese de aumento da interação água-rocha com a profundidade, possivelmente através do processo de ultrafiltração da água por argilas compactadas, agindo como membranas semipermeáveis.

\section{AGRADECIMENTOS}

Os autores agradecem à Petrobras, pelo financiamento e disponibilidade de amostras. Aos Geólogos Bruno Matos e Michael Strugale, integrantes da UN-Ba, pelo apoio técnico e pelas sugestões e correções feitas ao decorrer do trabalho e também aos revisores da RBGf pelos valiosos comentários e sugestões. 0 primeiro autor agradece à CAPES e ao CNPq, pela concessão da bolsa de mestrado.

\section{REFERÊNCIAS}

ARCHER JS \& WALL CG. 1994. Petroleum Engineering: Principles and Practice. Graham and Trotman, London. $350 \mathrm{p}$.

BOURG C, STIEVENARD M \& JOUZEL J. 2001. Hydrogen and oxygen isotopic composition of aqueous salt solutions by gas-water equilibration method. Chemical Geology, 173: 331-337.

BRAND WA, AVAK H, SEEDORF R, HOFMANN DE \& CONRADI T. 2000. New methods for fully automated isotope ratio determination from hydrogen at the natural abundance level. Geoph. Prosp., 28: 967-976.

BRUHN CHL \& DE ROS LF. 1987. Formação Sergi: evolução de conceitos e tendências na geologia de reservatórios. Boletim de Geociências da Petrobras, 1(1): 25-40.

CAIXETA JM, BUENO CV, MAGNAVITA LP \& FEIJÓ FJ. 1994. Bacias do Recôncavo, Tucano e Jatobá. Boletim de Geociências da Petrobras Estratigrafia das Bacias Sedimentares Brasileiras, 8(1): 163-172.

COPLEN TB \& HANSHAW BB. 1973. Ultrafiltration by a compacted clay membrane $-\mathrm{I}$. Oxygen and hydrogen isotopic fractionation. Geochimica et Cosmochimica Acta, 37: 2295-2310.

COSTE JF \& VALOIS JP. 2000. An Innovative Approach for the analysis of Production History in Mature Fields: A Key Stage for Field Reengineering. In: SPE Annual Technical Conference and Exhibition, Dallas, Texas. EUA. Disponível em: <http:/www.spe.org>. Acesso em: 22 fev. 2002. 
GAGLIANONE PC \& TRINDADE LAF. 1988. Caracterização Geoquímica dos Óleos da Bacia do Recôncavo. Geochimica Brasiliensis, 2(1): 1539.

GR00T PA. 2008. Handbook of Stable Isotope Analytical Techniques, Vol II, Elsevier, B.V., Oxford. 1398 p.

KOEHLER GD, CHIPLEY D \& KYSER TK. 1991. Measurement of the hydrogen and oxygen isotopic compositions of concentrated chloride brines and brines from fluid inclusions in halite. Chem. Geol. (Isot. Geosci. Sect.), 94: 45-54.

LEMAY TG. 2002. Sampling of Formation water from Wells in the Athabasca Oil Sands (in Situ) Area, Alberta, 1999-2001. A Compilation of Protocols and Methods; Albeta Energy and Utilities Board, EUB/AGS Geo-Note 11. 40 p.

LEVORSEN Al. 1958. Geology of Petroleum. Freeman and Company, INC, San Francisco. 703 p.

MILHOMEM PS, MAMAN EJ, OLIVEIRA FM, CARVALHO MS \& SOUZA LIMA W. 2003. Bacias Sedimentares brasileiras, Bacia do Recôncavo, Phoenix, 51.

MORAD S, WORDEN RH \& KETZER JM. 2003. Oxygen and hydrogen iso- tope composition of diagenetic clay minerals in sandstones: a review of the data and controlling parameters. In: WORDEN RH \& MORAD S (Eds.). Clay Mineral Cement in Sandstones. Int. Assoc. Sediment., Spec. Publ., 34: 63-91.

ROSTRON BJ \& HOLMDEN C. 2003. Regional variations in oxygen isotopic compositions in the Yeomanand Duperow aquifers, Williston basin (Canada-USA). Journal of Geochemical Exploration, 78-79: 337-341.

SEEWALD JS. 2003. Organic-inorganic interaction in petroleum producing sedimentary basins. Nature, 426: 327-333.

WEST AG, PATRICKSON SJ \& EHLERINGER JR. 2006. Water extraction times for plant and soil materials used in stable isotope analysis. Rapid Commun. Mass Spectrom., 20: 1317-1321.

WORDEN RH, MANNING DAC \& BOTTRELL SH. 2006. Multiple generations of high salinity formation water in the Triassic Sherwood Sandstone: Wytch Farm oilfield, onshore UK. Applied Geochemistry, 21: 455-475.

ZIEGLER K, COLEMAN ML \& HOWARTH RJ. 2001. Palaeohydrodynamics of fluids in the Brent Group (Oseberg Field, Norwegian North Sea) from chemical and isotopic compositions of formation waters. Applied Geochemistry, 16: 609-632.

\section{NOTAS SOBRE OS AUTORES}

Danilo Ribeiro de Sá Teles. Graduado em Física pela Universidade Federal da Bahia (UFBA). Atualmente é aluno de Pós-graduação do Centro de Pesquisa em Geofísica e Geologia (CPGG) da UFBA. Trabalhou no Departamento de Física da Terra e do Meio Ambiente, na mesma universidade, como Professor Substituto.

Antônio Expedito Gomes de Azevedo. Graduado como Bacharel em Física pela Universidade de São Paulo em 1963, e Ph.D. em Ciências da Terra pela Columbia University e Lamont-Doherty Geological Observatory em 1982. Atua desde 1965 como Professor do Instituto de Física da Universidade Federal da Bahia, atualmente é professor participante no Departamento de Física da Terra e do Meio Ambiente e no Curso de Pós-graduação em Geofísica. Vem trabalhando com isótopos estáveis, com interesse em Hidrologia Isotópica, Ciclo do Carbono, Paleoclimatologia e Caracterização isotópica de água de formação e de óleos de bacias petrolíferas.

Christian Pereira Lopes dos Santos. Possui graduação em Engenharia de Agrimensura pela Escola de Engenharia de Agrimensura da Bahia (2000), Mestrado e Doutorado na Universidade Federal da Bahia. Foi colaborador no Laboratório de Física Nuclear Aplicada (LFNA) e atualmente é professor do Instituto Federal Baiano. Tem experiência na área de Geociências, com ênfase em Geofísica Aplicada e Geofísica Nuclear (Pura e Ambiental), podendo atuar principalmente nos seguintes temas: Eletrorresistividade, Magnetometria, Hidrologia Isotópica, Hidrogeologia e Hidrogeoquímica. 